\title{
TU/e emonownen

\section{Two-dimensional mode I crack propagation in saturated ionized porous media using partition of unity finite elements}

\section{Citation for published version (APA):}

Kraaijeveld, F., Huyghe, J. M. R. J., Remmers, J. J. C., \& Borst, de, R. (2013). Two-dimensional mode I crack propagation in saturated ionized porous media using partition of unity finite elements. Journal of Applied Mechanics : Transactions of the ASME, 80, 020907-1/12. [020907]. https://doi.org/10.1115/1.4007904

DOI:

$10.1115 / 1.4007904$

Document status and date:

Published: 01/01/2013

\section{Document Version:}

Accepted manuscript including changes made at the peer-review stage

\section{Please check the document version of this publication:}

- A submitted manuscript is the version of the article upon submission and before peer-review. There can be important differences between the submitted version and the official published version of record. People interested in the research are advised to contact the author for the final version of the publication, or visit the $\mathrm{DOI}$ to the publisher's website.

- The final author version and the galley proof are versions of the publication after peer review.

- The final published version features the final layout of the paper including the volume, issue and page numbers.

Link to publication

\section{General rights}

Copyright and moral rights for the publications made accessible in the public portal are retained by the authors and/or other copyright owners and it is a condition of accessing publications that users recognise and abide by the legal requirements associated with these rights.

- Users may download and print one copy of any publication from the public portal for the purpose of private study or research.

- You may not further distribute the material or use it for any profit-making activity or commercial gain

- You may freely distribute the URL identifying the publication in the public portal.

If the publication is distributed under the terms of Article 25fa of the Dutch Copyright Act, indicated by the "Taverne" license above, please follow below link for the End User Agreement:

www.tue.nl/taverne

Take down policy

If you believe that this document breaches copyright please contact us at:

openaccess@tue.nl

providing details and we will investigate your claim. 


\title{
2D Mode I Crack Propagation in Saturated lonized Porous Media Using Partition of Unity Finite Elements.
}

\section{Olivier Coussy Memorial Special Issue}

\author{
F. Kraaijeveld* \\ Department of Biomedical Engineering \\ Eindhoven University of Technology \\ 5600MB Eindhoven, The Netherlands \\ Email: famkekraaijeveld@gmail.com
}

\begin{abstract}
J.M. Huyghe
Associate Professor, Member of ASME Department of Biomedical Engineering Eindhoven University of Technology 5600MB Eindhoven, The Netherlands

Email: j.m.r.huyghe@tue.nl
\end{abstract}

\section{J.J.C. Remmers}

Assistant Professor

Department of Mechanical Engineering Eindhoven University of Technology 5600MB Eindhoven, The Netherlands j.j.c.remmers@tue.nl

\section{R. de Borst}

Professor, Member of ASME Department of Mechanical Engineering Eindhoven University of Technology 5600MB Eindhoven, The Netherlands r.d.borst@tue.nl

Shales, clays, hydrogels and tissues swell and shrink under changing osmotic conditions, which may lead to failure. The relationship between the presence of cracks and fluid flow has had little attention, while the relationship between failure and osmotic conditions has had even less attention. The aim of this research is to study the effect of osmotic conditions on propagating discontinuities under different types of loads for saturated ionized porous media using the Finite
Element Method (FEM). Discontinuous functions are introduced in the shape functions of the FEM by partition of unity method, independently of the underlying mesh. The method allows for crack propagation in arbitrary directions. Damage ahead of the crack-tip is introduced by the cohesive zone model. Tensile loading of a crack in an osmoelastic medium results in opening of the crack and high pressure gradients between the crack and the formation. The fluid flow in the crack is approximated by Couette flow. Results show that 
depending on the load, permeability, prestress and the stiffness of the material, when crack propagation initiates, fluid is attracted to the crack-tip from the crack rather than from the surrounding medium causing the crack to close. The results show reasonable mesh-independent crack propagation for materials with a high stiffness. Interestingly, step-wise crack propagation through the medium is seen. This is because the propagation of the crack alternates with pauses in which the crack-tip area consolidates. As the load on the solid increases, the failure load is reached and the crack propagates again. Furthermore, propagation is shown to depend on the osmotic prestressing of the medium. This mechanism may explain the tears observed in intervertebral discs as degeneration progresses.

\section{Introduction}

Shales, clays, hydrogels and tissues swell and shrink under changing osmotic conditions. This change in conditions can lead to localized stresses and even to failure. This phenomenon is seen in the oil industry (e.g. hydraulic fracturing and borehole instability), in material design (clay, diapers, orthopaedic prosthesis and seals) and in medical biology (intervertebral disc herniation and tissue engineering). Examples of tensile failure are delamination, such as in the annulus of the intervertebral disc or in material test experiments, failure of concrete dams, pressurized well bores and hydraulic fracturing. In all these cases a strong coupling exists between fluid pressure and crack propagation. Numerical simulation techniques including coupling of fracture propagation and fluid exchange between the fracture and the formation are highly desirable to enhance the understanding of the process of tensile failure in these type of materials.

The etiology of intervertebral disc herniation is largely unknown. IVD tissue is avascular and therefore ages relatively fast [1]. During ageing, the disc changes in size, structure and osmotic prestress. Furthermore the number of cracks grow [1,2]. While some papers suggest that herniation is the result of weakening of the disc due to ageing [3-5], others believe that fast ageing is the cascading effect of an initial disruption [6,7]. Genetic studies actually suggest that occupation (i.e. loading history) is of less influence than genetics $[8,9]$. Wognum et al. [10] showed that cracks open under decreasing osmotic pressure (i.e. ageing) which causes stress localization at the crack-tip. The same mechanism may be responsible for borehole instability.

Some fluid-driven fracture propagation in ionized porous media, such as hydraulic fracturing, are desired. The controlled fracturing of tight sand is wanted in order to extract gas at commercial rates. Analytical solutions have been developed for the modeling of fluid-driven fractures [11-13]. More specifically, hydraulic fracturing in porous media $[14,15]$ is considered a benchmark. Detournay and Garagash [16] combined analytical and numerical analysis to show that fluid-driven fracture depends on permeability and propagation velocity for unsaturated porous media. Derivations of these solutions demanded simplifications, such as the assumption of no or minimal fluid exchange between crack and material.

In the past efforts have been made to simulate damage in porous media. Hogenization and upscaling techniques have been used $[17,18]$, but these are only suitable to study damage evolution when there is no prominent crack to be distinguished. Full fracture calculations for a macro crack in porous media were performed in a FE setting from the 1990s. Boone et al. [15] introduced interface elements equipped with the cohesive zone model (Dugdale [19] and Barenblatt [20]) for the fracturing process in a poro-elastic setting. They studied stress testing with water to initiate micro-cracking for a known path. The use of interface elements is suitable if the crack path is known a priori, since it requires a priori alignment of the element with the crack path. Adaptive remeshing methods have been combined with cohesive zone models $[21,22]$. These methods are able to capture fracture evolution in nonhomogeneous porous materials without assumptions about the singularities of the solid and fluid fields. Unfortunately, the adaptive remeshing schemes are computationally expensive and require suitable mapping procedures [22,23].

Alternatively, fractures are introduced in a mesh free way by exploiting the partition of unity property of the finite element shape functions [24]. Fractures are modeled as discontinuities in the displacement field. A discontinuity is introduced in the FEM by adding an enhanced (discontinuous) field to the standard displacement field. The number of degrees of freedom at the nodes whose support is crossed by a discontinuity, are increased. Therefore no new nodes are added during propagation and no dummy stiffness is required. Belytschko [25] introduced this method together with an asymptotic enhancement of the displacement field at the crack-tip. Practical benefits of exploiting the partition of unity method are that standard discretization is used and that crack propagation is independent of the discretization. The partition of unity approach has been combined in solid mechanics with cohesive zone models allowing the cohesive zone to run through the continuum elements [26-28].

The strong discontinuity concept has been introduced in porous media by Larsson et al. $[29,30]$ by the introduction of a regularized Dirac distribution function to the pressure field at the location of the discontinuity for shear banding in an enhanced strains approach, where Armero and Callari [31] assumed continuous flow.

Along the same lines, Roels et al. [32] used the partition of unity method to simulate crack propagation in the solid part combined with a moisture transfer 
scheme. Results show that the solution for the fluid phase smoothed upon mesh refinement, since a fine mesh is needed to capture the steep change increase of capillary pressure field and corresponding decrease in permeability. Al-Khoury et al. [33] used a strong discontinuity for the solid phase combined with a double porosity model for the fluid phase with coupling of fracture flow to the bulk material by a leakage term. Unfortunately only one-dimensional examples have been addressed. It is unclear how the model will behave in two dimensions.

Réthoré et al. $[34,35]$ used partition of unity approach with a strong discontinuity for the solid phase and a weak discontinuity for the fluid phase. Furthermore, fluid flow in the crack to the size of the opening is modeled by a cavity model assuming viscous fluid in the crack. Only static cases have been considered, but they show mesh-independency of the fluid phase.

All aforementioned papers have shown that the partition of unity approach is suitable for studying crack growth in porous media. While treatment of the solid phase is straightforward, there is no consensus on the treatment of the fluid flow as result of a displacement discontinuity, nor have osmotic forces been taken into account.

In this paper a framework for mesh-independent fracturing in ionized porous media under tensile loading is introduced. Lanir's plane strain osmoelastic model [36] for small deformations is used to model the bulk material. This model has as independent variables the displacement and the chemical potential of the fluid. It represents the bulk effect of the presence of ions, but assumes that the ion flow is fast compared to fluid diffusion.

The partition of unity approach is used to introduce strong discontinuities in the displacement field. The gradients in the pressure at fractures call for a weak discontinuity for the chemical potential field. This approach is combined with cohesive zone modeling [28] for damage evolution. A direct coupling between fluid flow in the crack, in the formation and between the formation and the crack, and coupling to ionization is formulated. Several examples are considered, both with and without an external mechanical load. The effect of prestress in the bulk material on fracture propagation is considered.

\section{Governing equations}

The governing equations consist of equations for the bulk and for the discontinuity, dominated by mass balance, momentum balance and constitutive behavior. A body $\Omega$ has an external boundary $\Gamma$ with a traction force on $\Gamma_{\mathrm{t}}$ and fluid supply on $\Gamma_{\mathrm{f}}$, with $\vec{n}$ the normal unit vector on the boundary $\Gamma$ directed outwards. The body is cut by a discontinuity $\Gamma_{d}$ in two domains, $\Omega^{+}$ and $\Omega^{-}$. The normal of the discontinuity $\vec{n}^{+}$is directed towards $\Omega^{+}$. Some constitutive behavior will introduce nonlinearity into the model. This is iteratively solved. The Newton-Rhapson procedure is used to calculate the results. Therefore, some elements are linearized.

\section{Bulk material}

Osmoelastic media have large negatively charged groups attached to the solid matrix. Counter charges are present in the fluid making the medium electrically neutral. Due to the fixed charges the total ion concentration inside the medium is higher than in the surrounding fluid. This excess of ion particles leads to an osmotic pressure difference, which causes swelling of the medium. The degrees of freedom for the model are the displacement $\vec{u}$ and the chemical potential of the fluid $\mu^{\mathrm{f}}$. Lanir's osmoelastic model [36] is summarized as (1).

(Momentum) $2 \mu \nabla^{2} \vec{u}+(c-2 \mu) \vec{\nabla} \operatorname{tr}\left(\boldsymbol{\epsilon}-\boldsymbol{\epsilon}_{i}\right)-\vec{\nabla} \mu^{\mathrm{f}}=0$
$\frac{\partial \operatorname{tr}\left(\boldsymbol{\epsilon}-\boldsymbol{\epsilon}_{i}\right)}{\partial t}-\vec{\nabla} \cdot\left(\boldsymbol{K} \vec{\nabla} \mu^{\mathrm{f}}\right)=0$,

with $c=2 \mu+\lambda+\frac{\partial \Delta \pi}{\partial \operatorname{tr}(\underset{\sim}{\epsilon})}$ with $\mu$ and $\lambda$ the Lamé constants and $\Delta \pi$ the osmotic pressure difference between the material and external salt solution. $K$ represents the permeability of the bulk material, in this case assumed isotropic and constant. The presence of ions fixed to the solid matrix results not only causes an osmotic pressure, but also prestressing of the material. Three types of prestress are considered (Fig. 1), namely the initial condition is the result of swelling in both directions (free swelling), in only $x$-direction or in only $y$-direction.

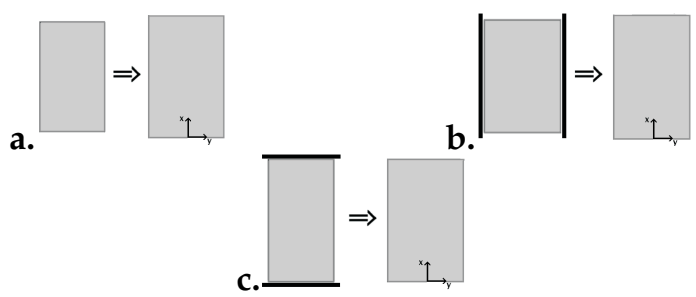

Fig. 1. Representation of three types of prestress: free swelling $\mathbf{a}$. in both directions, $\mathbf{b}$. in $x$-direction and $\mathbf{c}$. in $y$-direction.

\section{Discontinuity kinematics}

By exploiting the partition of unity principle [24], the displacement field and the chemical potential distribution is separated into two different scales: the bulk behavior (standard field) and crack behavior (enhanced field). The displacement field $\vec{u}$ of the body is 
additively decomposed into the standard or continuous part $\hat{\vec{u}}$ and the enhanced part $\tilde{\vec{u}}$.

$$
\vec{u}(\vec{x})=\hat{\vec{u}}(\vec{x}, t)+\mathcal{H}_{\Gamma_{d}}(\vec{x}) \tilde{\vec{u}}(\vec{x}, t)
$$

The Heaviside function $\mathcal{H}_{\Gamma_{d}}$ is defined by (3) with the jump at the middle of the discontinuity.

$$
\mathcal{H}_{\Gamma_{d}}= \begin{cases}+h / 2 & \vec{x} \in \Omega^{+} \\ -h / 2 & \vec{x} \in \Omega^{-}\end{cases}
$$

The Heaviside function is acting on a smooth function $\tilde{\vec{u}}(\vec{x}, t)$, hence allows for continuity of the crack surface. The jump at the discontinuity $\Gamma_{d}$ is given by $[\vec{u}]$ and represents the opening of the crack (shear or normal opening).

$$
[\vec{u}(\vec{x}, t)]=h \tilde{\vec{u}}(\vec{x}, t), \quad \vec{x} \in \Gamma_{d} .
$$

with $h=\mathcal{H}_{\Gamma_{d}}^{+}-\mathcal{H}_{\Gamma_{d}}^{-}$the magnitude of the jump of the Heaviside function.

Opening of a crack causes a high gradient in the chemical potential at both crack surfaces. In opening mode there is no pressure difference between the crack surfaces but a pressure difference between the crack surface and the middle of the crack. Using the partition of unity property, a distance function $\mathcal{D}_{\Gamma_{d}}$ similar to Réthoré et al. [34] is introduced. This function allows for the natural introduction of steep gradients without enforcing it. The decomposition becomes

$$
\mu^{\mathrm{f}}(\vec{x})=\hat{\mu}^{\mathrm{f}}(\vec{x}, t)+\mathcal{D}_{\Gamma_{d}}(\vec{x}) \tilde{\mu}^{\mathrm{f}}(\vec{x}, t)
$$

The distance function is defined with respect to the coordinates of the crack $\vec{x}_{\Gamma}$, namely by

$$
\mathcal{D}_{\Gamma_{d}}=\frac{h}{2}\left|\left(\vec{x}-\vec{x}_{\Gamma}\right) \cdot \vec{n}^{+}\right|, \quad \vec{x} \in \Omega
$$

with $h$ the magnitude of the Heaviside jump. The gradient of the distance function $\partial \vec{D}$ is given by

$$
\partial \overrightarrow{\mathcal{D}}=\nabla^{s} \mathcal{D}_{\Gamma_{d}}=\left\{\begin{array}{l}
\frac{h}{2} \vec{n}^{+} \vec{x} \in \Omega^{+} \\
\frac{h}{2} \vec{n}^{-} \vec{x} \in \Omega^{-}
\end{array}=\mathcal{H}_{\Gamma_{d}} \vec{n}^{+}\right.
$$

where $\nabla^{s} *=1 / 2\left\{\nabla *+(\nabla *)^{T}\right\}$ holds.

\section{Local behavior}

A local coordinate system $(n, s)$ is introduced where index $n$ denotes the normal component directed to $\Omega^{+}$ and $s$ denotes the tangential component directed towards direction of propagation. A displacement jump is decoupled into

$$
[\vec{u}]=[u]_{n} \vec{n}^{+}+[u]_{s} \vec{t}^{+}
$$

with $\vec{n}^{+}=-\vec{n}^{-}$directed into the body and $\vec{t}^{+}$is directed along the crack surface in direction of propagation. We define crack surfaces $\Gamma^{+}=\partial \Omega^{+} \cap \Gamma_{d}$ and $\Gamma^{-}=\partial \Omega^{-} \cap \Gamma_{d}$.

\section{Local mass balance}

Deformation around the discontinuity is strongly linked to fluid flow. Fluid flow takes place at the surface of the discontinuity from the medium into the crack and a flow along the crack when opening of the crack increases. When the crack is closed, the normal fluid flow $f^{ \pm}$over surface $\Gamma_{d}^{ \pm}$determines the amount of fluid exchange. When the crack opens, additional terms are included. Figure 2 shows a schematic overview of the local mass balance. In case of tensile

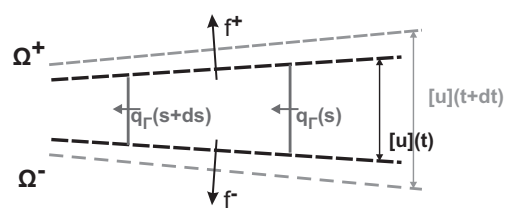

Fig. 2. Schematic representation of the fluid flow at the crack surface with parameter $S$ the distance along the crack.

mode, there is a balance between tangential flow and normal flow.

$$
\begin{aligned}
& f^{+}+f^{-}=-\frac{\partial q_{\Gamma}}{\partial s}-[\dot{u}]_{n}, \\
& q_{\Gamma} \quad=-\left|[u]_{n}\right|^{k} K_{d} \frac{\partial \mu_{\Gamma}^{\mathrm{f}}}{\partial s} .
\end{aligned}
$$

where $s$ represents the distance along the crack, with $s=0$ the crack-tip and $s$ positive in direction of $\vec{t}^{+}$. Tangential flow is assumed Couette flow in which $K_{d}$ is the permeability and $k$ a material factor.

\section{Local momentum balance}

A discrete crack is preceded by local damage. This micro-damage can be lumped into one constitutive relation (cohesive zone) and projected on the crack [28]. The model relates the decohesion, i.e. softening of the traction forces, as result of opening of the crack. As the damage is related to solid part of the material, the damage is related to the effective stresses.

At each crack surface, the following relation holds

$$
\left(\sigma_{\mathrm{e}}-\left(\mu^{\mathrm{f}}+\Delta \pi\right) \boldsymbol{I}\right)^{ \pm} \cdot \vec{n}^{ \pm}=\vec{t}_{\Gamma}^{ \pm}(\vec{x}, t), \vec{x} \in \Gamma^{ \pm}
$$




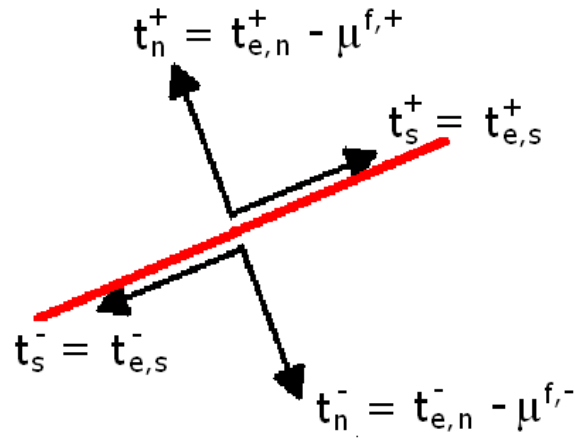

Fig. 3. Schematic representation of the traction forces at the crack surface

This is represented in Fig. 3. The cohesive law holds locally and is described in the local coordinate system of the discontinuity. The cohesive zone model is nonlinear. The change in traction forces is related to the displacements.

$$
\Delta t_{\Gamma}^{+}=\underline{T}_{\mathrm{e}} \underset{\sim}{\vec{u}}-\mu_{\Gamma}^{f} \underline{\sim}^{+}
$$

where $\underline{T}_{\mathrm{e}}$ the tangent stiffness containing the softening behavior. Furthermore, for the osmotic forces in the crack surfaces holds that the ionic concentration in the crack is the same as in the external reservoir.

$$
\mu_{\Gamma}^{f}=p_{\Gamma}-2 R T c^{\mathrm{ex}}, \Delta \pi^{+}=0,
$$

with $p_{\Gamma}$ hydraulic pressure in the crack.

\section{Yield criterion}

Crack growth is determined by the stress state in the solid matrix. Therefore the yield criterion, next to the cohesive zone, is related to the effective stress. The effective stress at the crack-tip varies locally and therefore the critical effective stress state is calculated nonlocally from the stress states around the crack-tip. A length scale $l_{a}$ is introduced which determines the influence radius and steepness of surrounding stress states.

To take the difference between compressive and tensile forces in to account, the yield law by Remmers et al. [28] is used. For that reason an equivalent traction $t_{\text {eq }}(\alpha)$, with $\alpha$ the to be determined angle, is introduced such that

$$
t_{\mathrm{e}, \mathrm{eq}}(\alpha)=\sqrt{<t_{\mathrm{e}, \mathrm{n}}(\alpha)>^{2}+\frac{1}{\beta}<t_{\mathrm{e}, \mathrm{s}}(\alpha)>^{2}}
$$

with $\langle x\rangle$ McCauley brackets, i.e. $x=0$ if $x<0$. $\beta$ is typically set to 2.3 [37] and the angle is limited to $0<\alpha<\pi$. Crack propagation is initiated when the equivalent traction force $\left(t_{\text {eq }}\right)$ is larger than the critical value $t_{u l t}$.

\section{Numerical description}

The weak form for the Finite Element Method is derived by standard Galerkin approach. Then the weak equations are discretized leading to a timedependent, non-linear system. This is solved using a Cranck-Nicholson scheme for time-integration and Newton-Rhapson iteration within each time increment.

\section{Weak form}

The momentum balance and mass balance are multiplied by test functions and subsequently integrated over the domain $\Omega$. These test functions are taken of the same form as the shape functions for the independent variables and therefore consisting of a continuous and discontinuous part. The displacements are enhanced with a strong discontinuity function. The chemical potential is enhanced with a weak discontinuity function.

$$
\vec{\eta}=\hat{\vec{\eta}}+\mathcal{H}_{\Gamma_{d}} \tilde{\vec{\eta}}, \varphi=\hat{\varphi}+\mathcal{D}_{\Gamma_{d}} \tilde{\varphi}
$$

Using integration by parts the discontinuity is introduced as a boundary integral. This is elaborated at the specific balance equations in the next section.

\section{Momentum balance}

The momentum equation after applying integration by parts is given by

$$
\begin{aligned}
& \int_{\Omega^{+} \cup \Omega^{-}} \nabla^{s}\left(\hat{\vec{\eta}}+\mathcal{H}_{\Gamma_{d}} \tilde{\vec{\eta}}\right): \sigma d \Omega=\int_{\Gamma_{\mathrm{t}}}\left(\hat{\vec{\eta}}+\mathcal{H}_{\Gamma_{d}} \tilde{\vec{\eta}}\right) \cdot \vec{t}_{\mathrm{t}} d \Gamma \\
& -\int_{\Gamma_{d}^{+}}\left(\hat{\vec{\eta}}+\mathcal{H}_{\Gamma_{d}} \tilde{\vec{\eta}}\right) \cdot \vec{t}_{\Gamma}^{+} d \Gamma-\int_{\Gamma_{d}^{-}}\left(\hat{\vec{\eta}}+\mathcal{H}_{\Gamma_{d}} \tilde{\vec{\eta}}\right) \cdot \vec{t}_{\Gamma}^{-} d \Gamma
\end{aligned}
$$

with $\sigma=\sigma_{\mathrm{e}}-p \boldsymbol{I}, p=\mu^{\mathrm{f}}+\Delta \pi$.

This equation must hold for all variations of test functions, therefore it holds also if $\hat{\vec{\eta}}=\overrightarrow{0}$ or if $\tilde{\vec{\eta}}=\overrightarrow{0}$. Both options are separately applied. This results in two equations. The first equation denotes the standard or continuous field behavior (taking $\tilde{\vec{\eta}}=\overrightarrow{0}$ and momentum balance demands $\vec{t}_{\Gamma}^{+}=-\vec{t}_{\Gamma}^{-}$). Then in total the next momentum balance for continuous field is found

$$
\int_{\Omega^{+} \cup \Omega^{-}} \nabla^{s} \hat{\vec{\eta}}: \sigma d \Omega=\int_{\Gamma_{\mathrm{t}}} \hat{\vec{\eta}} \cdot \vec{t}_{\mathrm{t}} d \Gamma
$$

The second equation denotes the behavior of the enhanced field (taking $\hat{\vec{\eta}}=\overrightarrow{0}$ ). With $t_{\Gamma}^{ \pm}=\vec{t}_{\mathrm{e}}^{ \pm}-\mu_{\Gamma}^{\mathrm{f}} \vec{n}^{ \pm}$, the momentum balance for the enhanced field is:

$$
\begin{aligned}
& \int_{\Omega^{+} \cup \Omega^{-}} \mathcal{H}_{\Gamma_{d}} \nabla^{s} \tilde{\vec{\eta}}: \sigma d \Omega+\int_{\Gamma_{d}^{+}} \tilde{\bar{\eta}} \cdot h\left(\vec{t}_{\mathrm{e}}^{+}-\mu_{\Gamma}^{\mathrm{f}} \vec{n}^{+}\right) d \Gamma \\
& =\int_{\Gamma_{\mathrm{t}}} \mathcal{H}_{\Gamma_{d}} \tilde{\bar{\eta}} \cdot \vec{t}_{\mathrm{t}} d \Gamma
\end{aligned}
$$




\section{Mass balance}

The variational mass balance is given by

$$
\begin{aligned}
& \int_{\Omega^{+} \cup \Omega^{-}} \nabla\left(\hat{\varphi}+\mathcal{D}_{\Gamma_{d}} \tilde{\varphi}\right) \cdot \vec{q} d \Omega \\
& -\int_{\Omega^{+}}\left(\hat{\Omega^{-}}\left(\hat{\varphi}+\mathcal{D}_{\Gamma_{d}} \tilde{\varphi}\right) \nabla \cdot \overrightarrow{\vec{u}} d \Omega\right. \\
& =\int_{\Gamma_{\mathrm{f}}}\left(\hat{\varphi}+\mathcal{D}_{\Gamma_{d}} \tilde{\varphi}\right) f_{\mathrm{f}} d \Gamma-\int_{\Gamma_{d}^{+}}\left(\hat{\varphi}+\mathcal{D}_{\Gamma_{d}} \tilde{\varphi}\right) \tilde{\vec{q}} \cdot \vec{n}^{+} d \Gamma \\
& -\int_{\Gamma_{d}^{-}}\left(\hat{\varphi}+\mathcal{D}_{\Gamma_{d}} \tilde{\varphi}\right) \tilde{\vec{q}} \cdot \vec{n}^{-} d \Gamma
\end{aligned}
$$

Taking $\tilde{\varphi}=0$, the mass balance for the continuous field becomes

$\int_{\Omega^{+} \cup \Omega^{-}} \nabla \hat{\varphi} \cdot \vec{q} d \Omega-\int_{\Omega^{+} \cup \Omega^{-}} \hat{\varphi} \nabla \cdot \dot{\vec{u}} d \Omega+\int_{\Gamma_{d}^{+}} \hat{\varphi} \tilde{\vec{q}} \cdot \vec{n}^{+} d \Gamma$ $+\int_{\Gamma_{d}^{-}} \hat{\varphi} \tilde{\vec{q}} \cdot \vec{n}^{-} d \Gamma=\int_{\Gamma_{\mathrm{f}}} \hat{\varphi} f_{\mathrm{f}} d \Gamma$

For the flow around the crack holds (9). In variational form this reduces to:

$$
\begin{aligned}
& \int_{\Gamma_{d}^{+}} \hat{\varphi} \tilde{\vec{q}} \cdot \vec{n}^{+} d \Gamma+\int_{\Gamma_{d}^{-}} \hat{\varphi} \tilde{\vec{q}} \cdot \vec{n}^{-} d \Gamma= \\
& =-\int_{\Gamma_{d}} \hat{\varphi} \frac{\partial q_{\Gamma}}{\partial s} d \Gamma-\int_{\Gamma_{d}^{+}} \hat{\varphi}[\overrightarrow{\vec{u}}] \cdot \vec{n}^{+} d \Gamma
\end{aligned}
$$

The term with tangential flow is simplified using divergence theorem

$$
\int_{\Gamma_{d}} \hat{\varphi} \frac{\partial q_{\Gamma}}{\partial s} d \Gamma=-\int_{\Gamma_{d}} \frac{\partial \hat{\varphi}}{\partial s} q_{\Gamma} d \Gamma+\hat{\varphi} q_{\Gamma} \mid s_{d}
$$

Combining Eqs. (20) and (21) with(19), the mass balance for continuous field is found:

$$
\begin{aligned}
& \int_{\Omega^{+} \cup \Omega^{-}} \nabla \hat{\varphi} \cdot \vec{q} d \Omega-\int_{\Omega^{+} \cup \Omega^{-}} \hat{\varphi} \nabla \cdot \dot{\vec{u}} d \Omega \\
& +\int_{\Gamma_{d}} \frac{\partial \hat{\varphi}}{\partial s} q_{\Gamma} d \Gamma-\int_{\Gamma_{d}} \hat{\varphi}[\overrightarrow{\vec{u}}] \cdot \vec{n}^{+} d \Gamma=\int_{\Gamma_{\mathrm{f}}} \hat{\varphi} f_{\mathrm{f}} d \Gamma+\left.\hat{\varphi} q_{\Gamma}\right|_{S_{d}}
\end{aligned}
$$

Using $\hat{\varphi}=0$ and $\mathcal{D}_{\Gamma_{d}}=0$ on $\Gamma_{d}$, the mass balance for the enhanced field is equal to

$$
\begin{aligned}
& \int_{\Omega^{+} \cup \Omega^{-}} \nabla\left(\mathcal{D}_{\Gamma_{d}} \varphi\right) \cdot \vec{q} d \Omega-\int_{\Omega^{+} \cup \Omega^{-}}\left(\mathcal{D}_{\Gamma_{d}} \varphi\right) \operatorname{tr}(\dot{\boldsymbol{\epsilon}}) d \Omega \\
& =\int_{\Gamma_{\mathrm{f}}}\left(\mathcal{D}_{\Gamma_{d}} \varphi\right) f_{\mathrm{f}} d \Gamma
\end{aligned}
$$

\section{Spatial discretization}

The weak form is provided by equations (16), (17), (22) and (23). Discretized forms are derived by dividing body $\Omega$ into elements $\Omega_{\mathrm{e}}, e=1 . . n_{\mathrm{e}}\left(\Omega=\bigcup_{1}^{n_{\mathrm{e}}} \Omega_{\mathrm{e}}\right)$. The result is that the discontinuity is discretized in elements $S_{d}$ and the boundary in elements $S_{\mathrm{e}}$. The displacements, the chemical potential and their variations are discretized similarly (Bubnov-Galerkin approach) by

$$
\begin{array}{ll}
\hat{\vec{u}}=\vec{N}^{T} \vec{a}_{u}, & \tilde{\vec{u}}=\vec{N}^{T} \vec{b}_{u} \\
\hat{\mu}^{\mathrm{f}}=\tilde{\sim}_{\sim}^{T}{ }_{\sim}^{\sim} \sim_{\mu}, & \tilde{\mu}^{\mathrm{f}}=\tilde{\sim}^{T} \underline{\sim}_{\mu}
\end{array}
$$

where $\underset{\sim}{\mathbb{N}}=\left[\begin{array}{ll}N_{\sim} & N_{\sim}\end{array}\right]$ contains the shape functions, which are the same in $x$ - and $y$-direction. The columns $\vec{a}_{u}$ and $\vec{b}_{u}$ contain the nodal values for bulk part and enhanced part, resp.. Similar are $\underset{\sim}{m}, a_{\mu}$ and ${\underset{\sim}{\mu}}_{\mu}$ columns of shape functions and nodal values. Then strain is discretized by

$$
\nabla^{s} \boldsymbol{\epsilon}=\underline{B} \underline{\vec{a}}_{u}+\mathcal{H}_{\Gamma_{d}} \underline{B}_{\sim} \vec{b}_{u}
$$

The first order differential operator on the displacement shape functions is denoted by $\underline{B}=\left[\underline{L} N_{x} L_{\sim} N_{y}\right]$ The matrix $L$ contains the differential operators:

$$
\underline{L}=\left(\begin{array}{cc}
\frac{\partial}{\partial x} & 0 \\
0 & \frac{\partial}{\partial y} \\
\frac{\partial}{\partial y} & \frac{\partial}{\partial x}
\end{array}\right)
$$

Furthermore $\underline{C}=\underline{L}^{T}\left(\vec{l} m^{T}\right)$ a $2 \times m$ matrix is defined with $\vec{l}=\left(\begin{array}{lll}1 & 1 & 0\end{array}\right)^{T}$. Then $\underline{B}^{T} \cdot\left(\vec{l}_{\sim} \underline{\sim}^{T}\right)=\vec{\sim} \underline{C}$ holds and $\nabla \mu^{\mathrm{f}}=C \underline{C}_{\mu}+\mathcal{H}_{\Gamma_{d}} \underline{C} b_{\mu}$.

Finally the stress and flux are discretized. The stress consist of the effective stress and the chemical potential. The effective stress in vector notation is given by $\sigma_{\tilde{e}}=\left(\sigma_{\mathrm{e}, x \mathrm{x}}, \sigma_{\mathrm{e}, y \mathrm{y}}, \sigma_{\mathrm{e}, \mathrm{xy}}\right)^{T}$. The stress is linearized with respect to the column of nodal values such that a Newton-Rhapson scheme can be used.

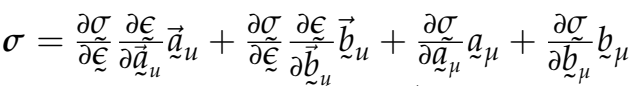

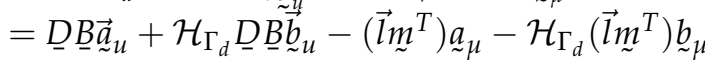

Here $\underline{D}$ is a $(3 \times 3)$ matrix for 2-D. The matrix $\underline{D}$ is defined as follows $\left(c=2 \mu+\lambda+\frac{\partial \Delta \pi}{\partial \operatorname{tr}(\underset{\sim}{)})}\right)$.

$$
\underline{D}=\left(\begin{array}{ccc}
c & (c-2 \mu) & 0 \\
(c-2 \mu) & c & 0 \\
0 & 0 & 2 \mu
\end{array}\right)
$$

The traction forces at the discontinuity $S_{d}$ are discretized as

$$
\left(\sigma_{\mathrm{e}}-\left(\mu^{\mathrm{f}}+\Delta \pi\right) \boldsymbol{I}\right) \cdot \vec{n}^{+}=\vec{t}_{\Gamma}^{+}=h \underline{\underline{T}} \vec{\sim}_{\sim}^{T} \vec{\sim}_{u}-\vec{n}^{+} \underline{\sim}^{T}{ }_{\sim} \mu
$$

with $\underline{T}_{\mathrm{e}}=Q \underline{T}_{\mathrm{e}, \mathrm{d}} Q$ the tangent stiffness of the cohesive zone. The seepage flux is linearized by

$$
\begin{aligned}
& \Delta \vec{q}=\frac{\partial \vec{q}}{\partial \overrightarrow{\vec{a}}_{u}} \cdot \vec{\sim}_{u}+\frac{\partial \vec{q}}{\partial \vec{b}_{\mu}} \cdot{\underset{\sim}{\vec{b}}}_{u}+\frac{\partial \vec{q}}{\partial a_{\mu}} a_{\mu}+\frac{\partial \vec{q}}{\partial \underline{\sim}_{\mu}} \underline{b}_{\mu} \\
& =-\underline{K} \underline{\underline{C}} \underline{a}_{\mu}-\tilde{\underline{K}}\left(\mathcal{D}_{\Gamma_{d}} \underline{C}+\partial \overrightarrow{\mathcal{D}}_{\Gamma_{d} m^{T}}\right) \underline{b}_{\mu}
\end{aligned}
$$


Finally, linearization of the local mass elements, results in the next elements.

$$
\begin{aligned}
& \int_{S_{d}^{+}} \frac{\partial \varphi}{\partial \eta} \vec{q}_{\Gamma} \cdot \vec{t}^{+} d \Gamma \\
& =\int_{S_{d}^{+}} \frac{\partial \dot{m}}{\partial \tilde{\eta}}\left|\left[\vec{u}_{\mathrm{i}}\right]^{T} \vec{n}^{+}\right|{ }^{k} K_{d} \frac{\partial m^{T}}{\partial \tilde{\eta}} d \Gamma a_{\mu} \\
& +\int_{S_{d}^{+}} \frac{\partial m}{\partial \eta} K_{d} \frac{\partial \mu_{\mathrm{i}}^{\mathrm{f}}}{\partial \eta} k\left|\left[\vec{u}_{\mathrm{i}}\right]^{T} \vec{n}^{+}\right|^{k-1} \operatorname{sgn}\left(\left[\vec{u}_{\mathrm{i}}\right] \cdot \vec{n}^{+}\right)\left(\vec{n}^{+}\right)^{T} \vec{N}^{T} d \Gamma \overrightarrow{\underline{b}}_{u}
\end{aligned}
$$

$$
\left.\varphi \vec{q}_{\Gamma} \cdot \vec{t}^{+}\right|_{S_{d}^{+}}=\left.\underset{\sim}{m} \vec{q}_{\Gamma} \cdot \vec{t}^{+}\right|_{S_{d}^{+}}
$$

\section{Time discretization}

To solve the system of equations, a time stepping algorithm is required. The solution is sensitive to the magnitude of the time increment (time step). A large step leads to under estimation of fluid pressure in confined compression. Taking too small steps leads to initial oscillation. For stable time integration it is required that [38]:

$$
\Delta t>\frac{\Delta x^{2}}{c K}
$$

This means that the time needed for the fluid to propagate a distance $\Delta x$ is related to the the square of the distance, permeability of the framework and the stiffness of the framework.

In this model the Cranck-Nicholson scheme is used. This means that differential terms are approximated linearly and weighted. Stabilization is reached if implicit time stepping is used.

\section{Total system}

The total set of governing equations and discontinuity equations is given by

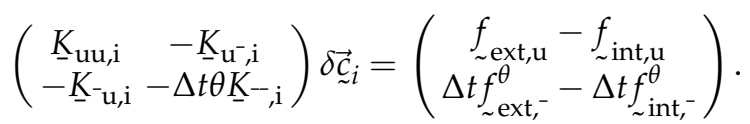

where $\vec{c}_{i}$ contains the nodal values of the displacements and chemical potential for the continuous and discontinuous part. In the equations subscript $i$ denotes the temporary solution $\vec{c}_{i}$ in iteration step $i$ and superscript $n$ denotes the solution $\vec{c}^{n}$ of previous time step $n$. Even though matrices $\underline{D}, \underline{K}, \underline{T}_{\mathrm{e}}$ are symmetric, $\underline{M}_{\mu u, i}$ and $\underline{M}_{u \mu, i}$ cause asymmetry of the system.

The solution procedure is driven by time. In the examples the displacement is increased monotonically. Snap-back can occur due to non-uniqueness of the solution.

The model has been programmed using the Jem/Jive finite element toolkit which has been developed by Habanera, B.V., Delft, The Netherlands. For implementation aspects like the tracking of the crack-tip, increasing the degrees of freedom or other propagation issues we refer to Remmers et al. [28,39,40].

\section{Numerical examples}

In the next examples an exponential cohesive law is used for the damage evolution, i.e. softening behavior after reaching the critical stress state. Defined is critical length $\delta_{n}=\mathcal{G}_{c} / \tau_{u l t}$, with $\tau_{u l t}$ the ultimate traction forces and $\mathcal{G}_{c}$ the fracture toughness. The cohesive law is then for positive opening

$$
t_{n}=\tau_{u l t} e^{-\left(\frac{[u] n}{\delta_{n}}\right)},[u]_{n} \geq 0 .
$$

This is shown in Fig. 4. A history parameter $\kappa$ is in-

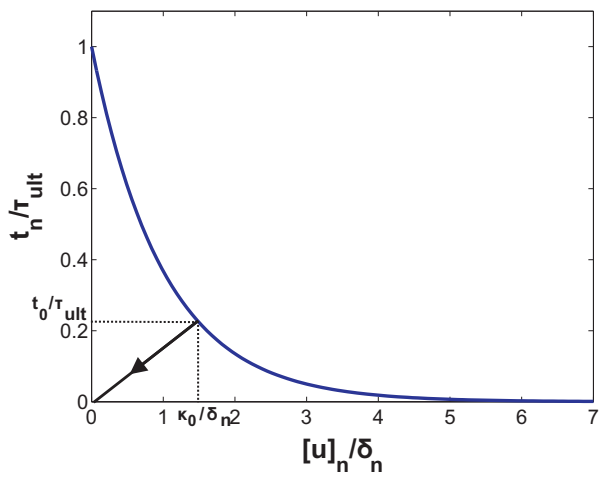

Fig. 4. Normalized distribution of the exponential cohesive law for tensile loading related traction forces and displacement.

troduced to incorporate unloading. Each time step the current opening $\kappa_{0}$ and traction $\tau_{0}$ are stored. When the new opening is smaller than previous, then unloading takes place according to

$$
t_{n}=\frac{\tau_{0}}{\kappa_{0}}[u]_{n}
$$

Damage is defined as

$$
D=1-\frac{t_{n}}{\tau_{u l t}}
$$

Macro crack is developed when the local damage approaches maximum $(D=1)$, i.e. when the cohesive law approaches zero. When locally the opening decreases compared to previous time step unloading takes place. The cohesive law parameters $\mathcal{G}_{c}$ and $\tau_{u l t}$ can be obtained from experimental data. The temperature is assumed $298 \mathrm{~K}$ in all examples. 


\section{Delamination test}

A delamination test is performed in which a crack propagates at a predefined angle of $0^{\circ}$. An initial defect of length $13 \mathrm{~mm}$ is inserted on the lefthandside, Fig. 5. The sample is fixed on the righthandside and is in contact with a filter $\left(\mu^{\mathrm{f}}=0\right)$. Crack propagation is initiated by pulling the sample on the top and bottom over approximate $8 \mathrm{~mm}$ with a fixed velocity of $1.0 \mathrm{e}-3 \mathrm{~mm} / \mathrm{s}$. The local fluid distribution is determined by Eq. 9 .

The material properties and boundary conditions

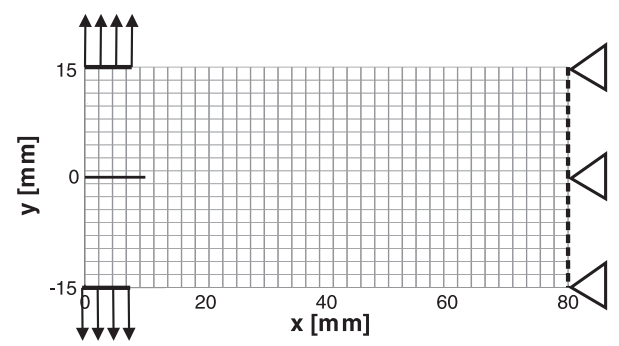

Fig. 5. The mesh and boundary conditions for delamination consisting of 575 elements. Material is pulled at the top and bottom on the left and is on the right in contact with a filter.

are given in table 1 . This means that $2 R T \Gamma c^{\mathrm{ex}}=$ $4.96 \mathrm{~N} / \mathrm{mm}^{2}$ holds. A time step of $0.1 \mathrm{~s}$ is used.

The influence of mesh refinement and time discretiza-

Table 1. Material properties and boundary conditions for delamination test.

\begin{tabular}{rlc}
\hline \hline$E$ & $=1.4 \mathrm{e}+4$ & {$\left[\mathrm{~N} / \mathrm{mm}^{2}\right]$} \\
$\varphi_{i}^{\mathrm{f}}$ & $=0.10$ & {$[-]$} \\
$c^{\mathrm{ex}}$ & $=1.0 \mathrm{e}-3$ & {$\left[\mathrm{mmol} / \mathrm{mm}^{3}\right]$} \\
$K_{d}$ & $=2.0 \mathrm{e}-1$ & {$\left[\mathrm{~mm}^{4} \mathrm{~N}^{-1} \mathrm{~s}^{-1}\right]$} \\
$\mathcal{G}_{c}$ & $=0.020$ & {$[\mathrm{~N} / \mathrm{mm}]$} \\
$l_{a}$ & $=7.8$ & {$[\mathrm{~mm}]$} \\
$v$ & $=0.33$ & {$[-]$} \\
$K$ & $=2.0 \mathrm{e}-1$ & {$\left[\mathrm{~mm}^{4} \mathrm{~N}^{-1} \mathrm{~s}^{-1}\right]$} \\
$c_{i}^{\mathrm{fc}}$ & $=-1.0 \mathrm{e}-3$ & {$\left[\mathrm{mmoleq}^{3} \mathrm{~mm}^{3}\right]$} \\
$\mathrm{k}$ & $=2$ & {$[-]$} \\
$\tau_{\text {ult }}$ & $=1.1$ & {$\left[\mathrm{~N} / \mathrm{mm}^{2}\right]$} \\
$v$ & $=1.0 \mathrm{e}-3$ & {$[\mathrm{~mm} / \mathrm{s}]$} \\
\hline
\end{tabular}

tion on the crack propagation and the flow around the crack is considered. For the mesh refinement, the mesh of Fig.5 is refined to 2701 elements. The corresponding time step is a quarter of the time step of the coarse mesh. Furthermore, the influence of local mass balance is considered by decreasing the local permeability with respect to the standard case or prescribing the chemical potential in the crack.

\section{Pull test}

In the second case a pull test is considered with a yield criterion based on optimal principal stress as mentioned in the model section. An initial defect of approximately $22 \mathrm{~mm}$ is inserted in the middle, Fig. 6 . The sample is fixed at the bottom and is pulled at the top with constant speed, while in contact with a filter at the sides $\left(\mu^{\mathrm{f}}=0\right)$. The material properties are identical to those specified in table 1 , except that $K_{d}=2.0 \mathrm{e}-1$ $\mathrm{mm}^{4} \mathrm{~N}^{-1} \mathrm{~s}^{-1}$ and $K=2.0 \mathrm{e}-1 \mathrm{~mm}^{4} \mathrm{~N}^{-1} \mathrm{~s}^{-1}$. Crack propagation is initiated by loading the sample over the complete width with a fixed velocity of $5.0 \mathrm{e}-4 \mathrm{~mm} / \mathrm{s}$. A time step of $d t=0.1 \mathrm{~s}$ is used. In this second case the

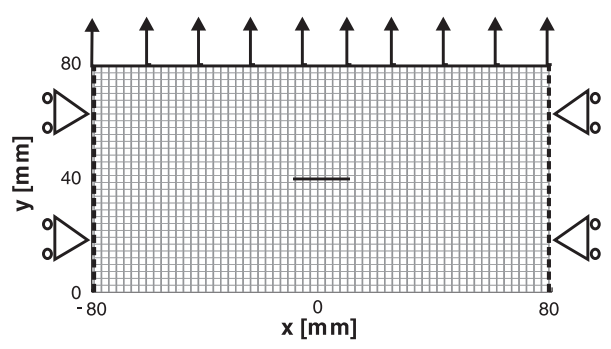

Fig. 6. The mesh and boundary conditions for pull test: material is pulled at the top with bottom fixed and at sides in contact with a filter.

angle of growth is not prescribed. To ensure growth only under tension, equivalent traction forces are used, with $\beta=2.3$, Eq. (13). Similarly to delamination, the influence of prestress and local mass balance is considered.

\section{Osmolarity test}

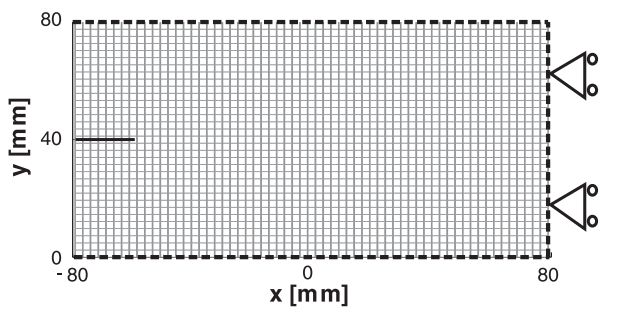

Fig. 7. The mesh and boundary conditions for the osmolarity test: the material is fixed at bottom, right and top and is in contact with a filter at these sides.

As a last example, a test is performed in which the effect of decreasing osmotic pressure is considered on a crack with length $\approx 18 \mathrm{~mm}$ at the rim of the material. For that purpose the external salt concentration is increased, i.e. the chemical potential on the boundaries 
Table 2. Material properties and boundary conditions for osmolarity test.

\begin{tabular}{lcc}
\hline \hline$E$ & $=90.0$ & {$\left[\mathrm{~N} / \mathrm{mm}^{2}\right]$} \\
$\varphi_{i}^{\mathrm{f}}$ & $=0.80$ & {$[-]$} \\
$c^{\mathrm{ex}}$ & $=0.15 \mathrm{e}-3$ & {$\left[\mathrm{mmol} / \mathrm{mm}^{3}\right]$} \\
$K_{d}$ & $=2.8 \mathrm{e}-4$ & {$\left[\mathrm{~mm}^{4} \mathrm{~N}^{-1} \mathrm{~s}^{-1}\right]$} \\
$\mathcal{G}_{c}$ & $=0.2 \mathrm{e}-2$ & {$[\mathrm{~N} / \mathrm{mm}]$} \\
$l_{a}$ & $=7.8$ & {$[\mathrm{~mm}]$} \\
$v$ & $=0.2$ & {$[-]$} \\
$K$ & $=2.8 \mathrm{e}-4$ & {$\left[\mathrm{~mm}^{4} \mathrm{~N}-1 \mathrm{~s}^{-1}\right]$} \\
$c_{i}^{\mathrm{fc}}$ & $=-0.2 \mathrm{e}-3$ & {$\left[\mathrm{mmoleq}^{\mathrm{mm}} \mathrm{mm}^{3}\right]$} \\
$\mathrm{k}$ & $=2$ & {$[-]$} \\
$\tau_{u l t}$ & $=0.25$ & {$\left[\mathrm{~N} / \mathrm{mm}^{2}\right]$} \\
$\Delta \mu^{\mathrm{f}}$ & $=-0.4 \mathrm{e}-3$ & {$\left[\mathrm{~N} / \mathrm{mm}^{2}\right]$} \\
\hline & &
\end{tabular}

is decreased. The material is fixed at the top, right side and bottom to a filter which alters the chemical potential by $-0.4 \mathrm{e}-3 \mathrm{~N} / \mathrm{mm}^{2}$.

$$
\Delta \mu^{\mathrm{f}}=\Delta \pi\left(c_{i}^{\mathrm{ex}}+\Delta c^{\mathrm{ex}}\right)-\Delta \pi\left(\Delta c^{\mathrm{ex}}\right) .
$$

This means that the external salt concentration is increased from initial state by $\Delta c^{\mathrm{ex}}=4.82 \mathrm{e}-7 \mathrm{t}$. A time step of $8.0 \mathrm{~s}$ is used. The material properties and conditions are given in table 2 .

The influence of prestress is considered on crack propagation and flow around the crack.

\section{Results \\ Delamination test}

The presence of fixed charges causes an initial strain of $\epsilon_{i, x}=\epsilon_{i, y}=1.9 \mathrm{e}-5$ in case of prestress in both direction and an initial strain of $2.8 \mathrm{e}-5$ when only prestressed in one direction. A weak interface criterion causes propagation in a straight line for all cases.

Considering the chemical potential distribution, Fig. 8, the figures show localization at the crack-tip with a negative chemical potential. This low chemical potential is relaxed by fluid redistribution towards the cracktip. The chemical potential is largest at the left due to largest opening of the crack.

In order to check the results, the spatial discretization is refined (from 575 to 2701 elements, "dx refine") and time discretization is refined without refining in space (from $0.5 \mathrm{~s}$ to $0.125 \mathrm{~s}$, "dt refine"). Results are shown approximately at the same point (Fig. 9). It is evident that both in time and in space, the results are the same, except for the slight mismatch in space due to a mismatch in integration points between the coarse and fine

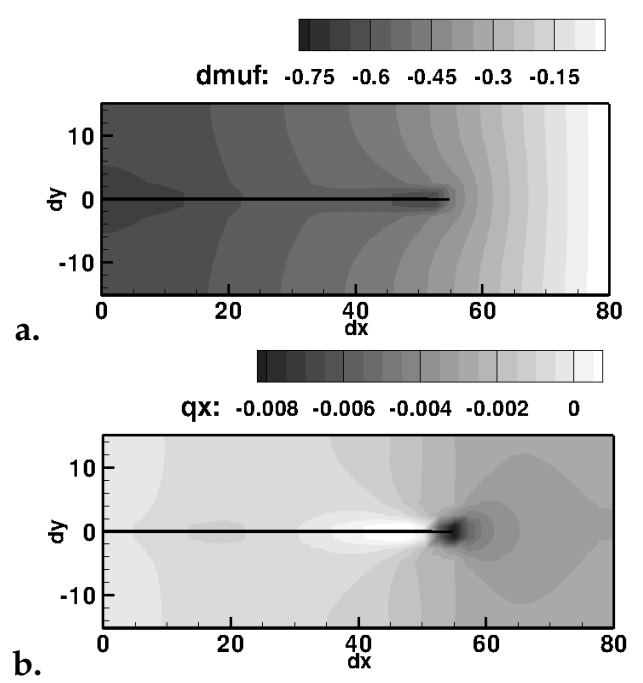

Fig. 8. Delamination in case of prestress in both directions after 950 time increments (i.e. $9.5 \mathrm{e}-2 \mathrm{~mm}$ displacement of top boundary). Distribution of a. chemical potential in $\left[\mathrm{N} / \mathrm{mm}^{2}\right]$ and $\mathbf{b}$. flow in $x$ direction in $[\mathrm{mm} / \mathrm{s}]$.

mesh.

Finally, the influence of local mass balance is consid-

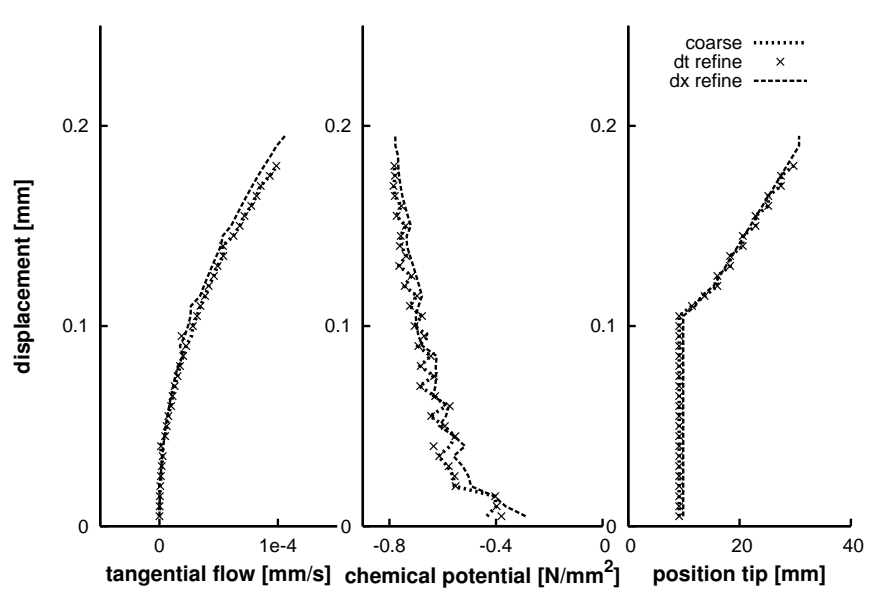

Fig. 9. Comparison of the effect mesh- and time refinement for delamination at a point just ahead of the initial crack $(d x=13.71 \mathrm{~mm})$.

ered. Decreasing the crack permeability decreases the tangential flow. It does not alter the crack path dramatically. When assuming contact with an external reservoir (i.e. $\mu_{\Gamma}^{f}=0$ ), the flow is reversed with respect to the previous cases. The fluid distribution (Fig. 10) shows that the chemical potential is now highest in front of the macro crack, instead of at the start of the macro crack such as in the previous cases.

\section{Pull test}

This case considers a more compliant material then for delamination. The prestress caused an initial strain 

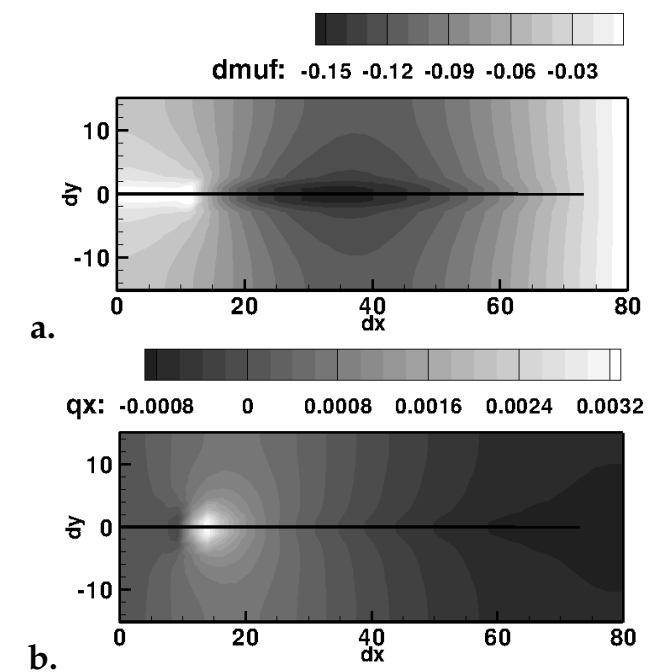

Fig. 10. Distribution of chemical potential in $\left[\mathrm{N} / \mathrm{mm}^{2}\right]$ for delamination in case of prestress in both directions and $\mu_{\Gamma}^{f}=0$ after 1100 increments (i.e. displacement of top boundary of $d u=1.1 \mathrm{e}-2 \mathrm{~mm}$ ) for $\mathbf{a}$. chemical potential and $\mathbf{b}$. flow in $x$-direction.

of $\epsilon_{i, x}=\epsilon_{i, y}=1.9 \mathrm{e}-4$ in case of prestress in both direction and an initial strain of $2.8 \mathrm{e}-4$ when prestressed in only one direction.

Figure 11 shows the chemical potential distribution and flow in case of prestress in both directions with crack growth. Even though the direction of crack growth is not pre-defined, the damage growth is almost straight. Localization takes place at both cracktips with low chemical potential, Fig. 11a. Fluid is attracted from the left and right towards the crack. The stress field is the largest in the middle of the crack (Fig. 11b).

The displacement, tangential flow and chemical potential are considered almost in the middle of the crack (Fig. 12). For all cases of osmotic prestress, the chemical potential becomes more negative, though in the case without prestress the chemical potential decreases less. In the case of prestress in $x$-direction only, a negative opening is seen at start and increases, which causes a wave in tangential flow. Furthermore, the time for crack to initiate increases. When damage initiates, a steep increase in chemical potential is seen in the middle of the crack. Unfortunately, Newton-Rhapson does not converge anymore after several growth stages.

Trends do not alter under mesh and time discretization refinement. Lastly, when changing the boundary condition at the crack to the boundary condition representing contact with a reservoir, resulted in oscillations in pressure and displacements.

\section{Osmolarity test}

The sample is subjected to an increase of external salt concentration. The result is a global decrease in chemical potential (Fig. 13a) and a local increase of stress ahead of the crack-tip (Fig. 13b).

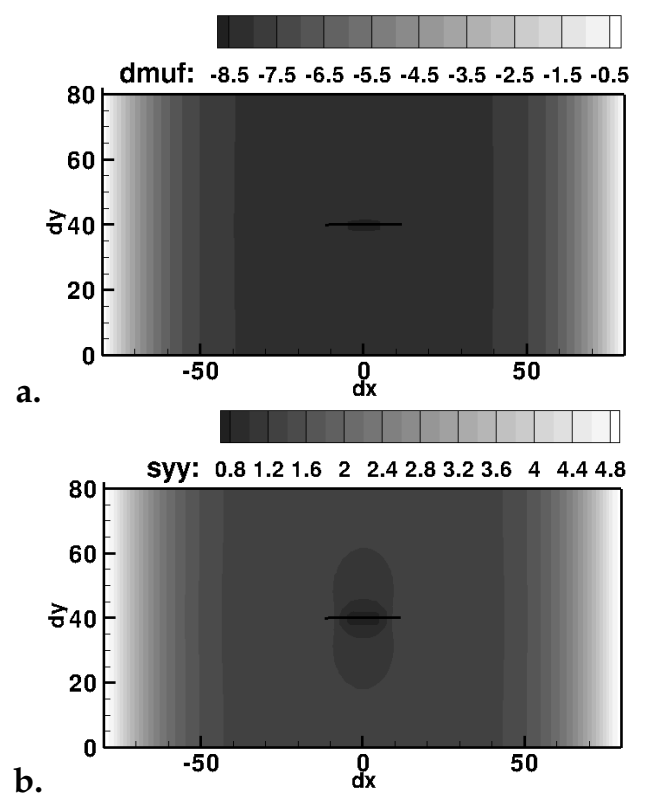

Fig. 11. Distribution for pull test of a. chemical potential and $\mathbf{b}$. stress component $\sigma_{\mathrm{e}, \mathrm{yy}}$ for the pull test after 1010 increments (i.e. $5.05 e-2 \mathrm{~mm}$ of pull displacement) in case of prestress in both directions including cohesive zone.

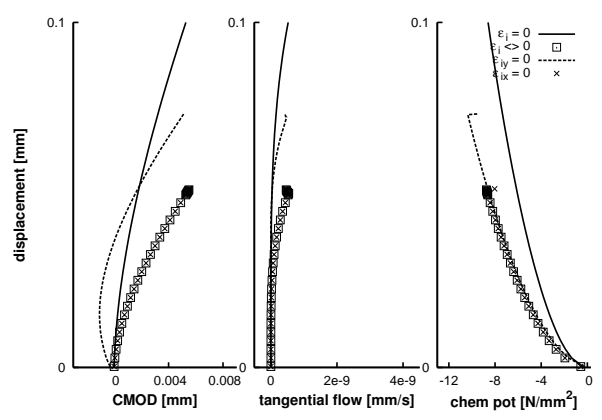

Fig. 12. Comparison for the pull test of the effect of prestress on crack mouth opening displacement (CMOD), chemical potential and tangential flow in the crack at $d x=3.5 \mathrm{~mm}$.

The flow is considered in a close-up of the cracktip (Fig. 14). Crack growth causes a local flow at the crack-tip directed towards the crack-tip (in $x$ - and $y$ direction), while the flow away from the crack-tip is directed opposite in the crack.

This trend is seen for all cases with prestress, except for osmotic prestress in only $x$-direction. In that case a solution could not be found. Of the other two cases, damage progressed longest in case of prestress only in the $y$-direction. Furthermore, in case of prestress in both directions, opening of the crack is largest while chemical potential in the crack is smallest. This pattern is seen throughout time. The fact that the curve for prestress in only $y$-direction is longer than in case of prestress in both directions, but with zero values, means that damage has occurred but not evolved. 


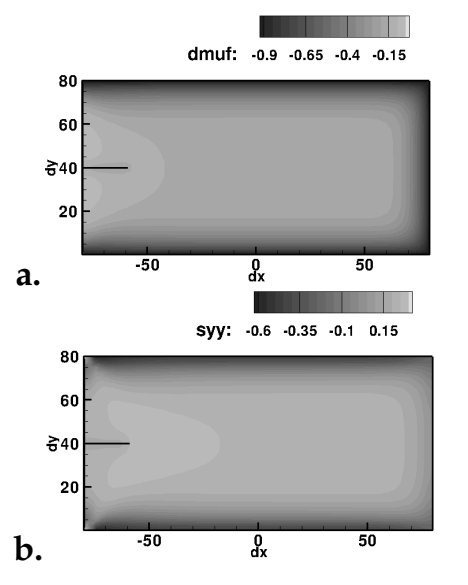

Fig. 13. Distribution for the osmolarity test after 300 increments (i.e. a change in chemical potential of $-0.96 \mathrm{MPa}$ ) just before damage initiation for prestress in both directions for $\mathbf{a}$. of chemical potential and $\mathbf{b}$. of stress component $\sigma_{\mathrm{e}, \mathrm{yy}}$.

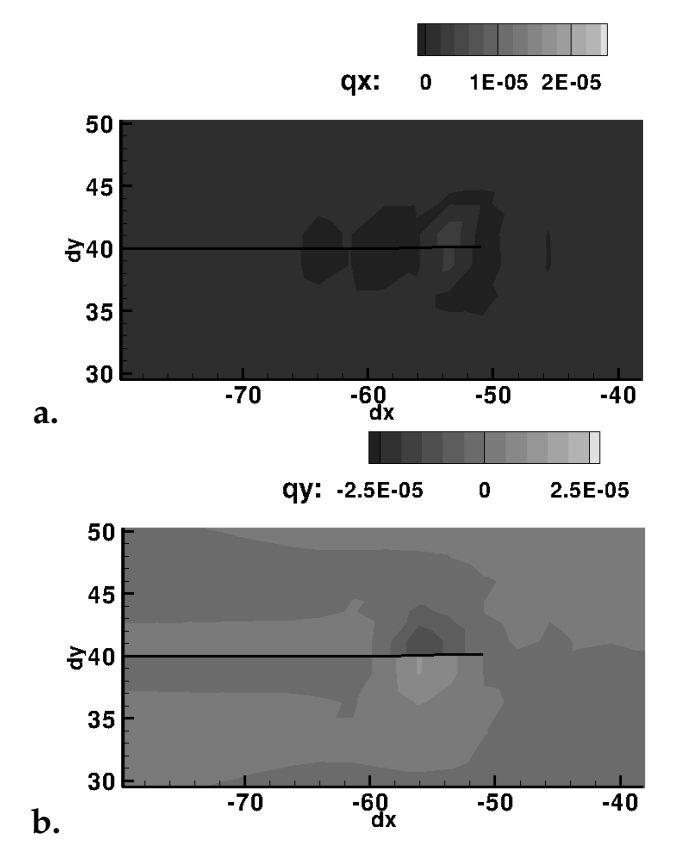

Fig. 14. Distribution at the crack-tip for osmolarity test of flow for prestress in both directions after 306 increments (i.e. a change in chemical potential of $-0.98 \mathrm{MPa}$ ) just after damage initiation $\mathbf{a}$. in $x$-directions and $\mathbf{b}$. $y$-direction.

\section{Discussion}

In this paper has addressed a small strain numerical model to study the interplay between prestressing of the solid material, fluid flow and mode-I crack propagation for ionized porous media. The partition of unity approach is used to integrate cracks into the FEM. A strong discontinuity is inserted in the displacement field by enhancing the field by the Heaviside function. A weak discontinuity is inserted in the field of chemical potential via a distance function to capture the high gradient in this field.

The results are meant to indicate trends rather than

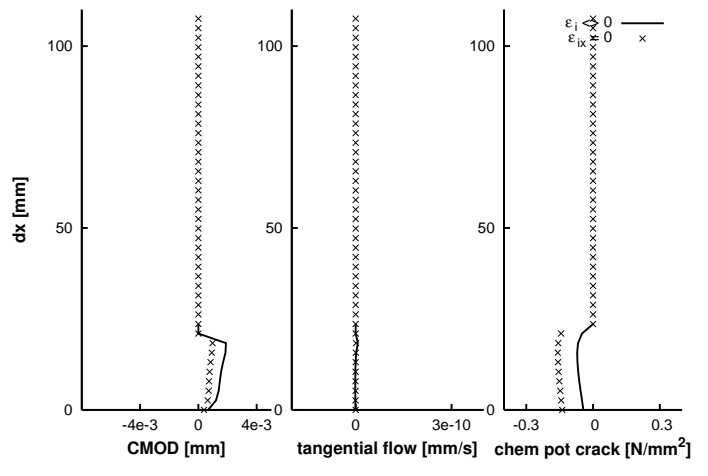

Fig. 15. Evolution for the osmolarity test in plane of crack of chemical potential, tangential flow and crack mouth opening (CMOD) after 130 increments, $\Delta \mu^{\mathrm{f}}=-0.4 M P a$.

yielding quantitative predictions. The model captures local trends well. A profound influence is seen of the fluid phase on the crack propagation in the solid. The presence of fluid slows down crack initiation and propagation. The flow from the surrounding medium causes an increase in volumetric strain at the cracktip, and hence an increase of the (tensile) effective stress at the crack-tip. This increase triggers damage and possible further crack propagation. Upon crack propagation a local change in flow direction is seen. The deformation around the crack causes fluid to flow mostly from the crack itself, because the permeability is far higher in the crack than in the medium. This flow from the crack to the crack-tip causes closing of the crack and, hence, slows down crack propagation. The expression of this phenomenon depends on loading protocol and on (direction) of prestress.

Considering the example without external mechanical load, opening and growth of the crack takes place due to a global decrease in osmotic pressure. This phenomenon is similar to the drying of clay, but in fully saturated conditions. This mechanism could be an explanation for the poor relation between intervertebral disc herniation and load. Furthermore this could be a mechanism for borehole instability.

Bilinear shape functions with chemical potential and displacements at the corner nodes are used to calculate the solution as a simplification. Réthoré et al. [34] show that these approximations are sufficient for structural response in non-propagating cracks when the mesh is fine enough and do not produce issues related to the Babuska-Brezzi conditions [41]. This condition requires a higher order interpolation of the displacement field than of the pressure field. The introduction of the distance function even increases the order of interpolation of the pressure field locally. This is needed for the higher order terms in the cavity model [34]. As the higher order of interpolation is used only locally, the order of integration does not seem to produce any stability problems related to the Babuska-Brezzi condition. Further comparison 
between this approach and literature is difficult since partially saturated conditions are considered $[32,33]$. Furthermore, results, such as mesh-dependency, is most prominent in lower stiffness cases.

Below the results are addressed in more detail. Generally, upon loading the effective stresses at the crack-tip increase and the chemical potential decreases. This is due to fluid flow away from the crack-tip. Upon crack propagation the fluid redistribution causes the effective stress at the tip to suddenly decrease and the chemical potential to increase. The time needed to resolve the flow over the extension is equivalent to the stiffness times permeability $\left(\Delta t \sim \Delta x^{2} / c K\right)$.

In case of the delamination test, the stiffness and permeability are so high that fluid (nor chemical potential nor tangential flow) hardly seems to influence crack growth. Furthermore, the presence of prestress seems to enhance crack propagation. The contact with a reservoir changes the flow pattern at the crack, but influence on crack propagation is low.

When the material is less stiff, the influence of the fluid becomes more profound. In the pull test, the chemical potential in the crack decreases to such an extent, that the osmotic pressure in the crack is lower than in the material. The low osmotic pressure in the crack causes flow from the material into the crack and with that further opening of the crack. The time to initiation is longer in case of no prestress than with prestress due to the slower opening of the crack (Fig. 12). In the case without initial prestress in $y$-direction, the crack closes temporarily due to the larger osmotic pressure in the bulk material than in the crack.

Upon crack growth a steep change in chemical potential takes place. Due to the lower stiffness and permeability of the material than the previous case, more time is needed to resolve the flow. Furthermore, the stress at the crack-tip is not relieved as it should be, which causes fast crack growth. Finally, the mismatch between the physical flow and the simulated flow via the weak discontinuity causes loss of numerical stability. A solution cannot be found anymore.

In the last case the material is not loaded mechanically at the external boundary of the material, but crack initiation is dominated by internal loading. A material of relatively low stiffness is considered. By changing the external salt concentration at the outside of the material, the osmotic pressure is lower outside than inside the material. This difference in osmotic pressure initiates flow. Flow is attracted from inside the crack near the crack-tip, rather than from the surrounding medium. The result is opening of the crack. The opening of the crack is largest in case minimal prestress in $y$-direction (Fig. 15), since the pressure difference between the material and the crack is larger. In all cases stability of the calculations is lost upon the forming of the macro crack, most probably because the fluid flow is unresolved.
Depending on the material properties, prestress can either enhance or slow done crack propagation. In case of very stiff materials, propagation can be enhanced by the extra stiffness. In lower stiffness cases, such as the intervertebral disc, the prestress provides protection against small osmotic changes. When ionization is lost, cracks can open again and propagate.

\section{Appendix}

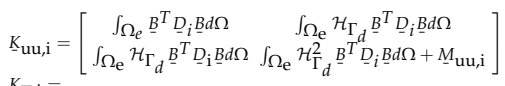

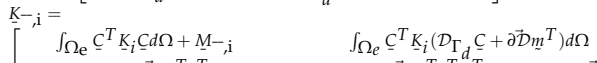

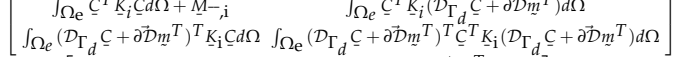

$$
\begin{aligned}
& K_{u \mu}=\left[\begin{array}{ccc}
\int_{\Omega_{\mathrm{e}}} \overrightarrow{\mathrm{N}} C d \Omega & \int_{\Omega_{\mathrm{e}}} \overrightarrow{\mathrm{N}}\left(\mathcal{D}_{\Gamma_{d}} \underline{C}+\partial \vec{D} m_{\underline{m}} T\right) d \Omega
\end{array}\right] \\
& \underline{K}_{u \mu}=\left[\begin{array}{lll}
\int_{\Omega_{\mathrm{e}}} \mathcal{H}_{\Gamma_{d}} \vec{N} C d \Omega+M_{u \mu, i} & \int_{\Omega_{\mathrm{e}}} \mathcal{H}_{\Gamma_{d}} \overrightarrow{\mathrm{N}}\left(\mathcal{D}_{\Gamma_{d}} \underline{C}+\partial \vec{D} m^{T}\right) d \Omega
\end{array}\right]
\end{aligned}
$$

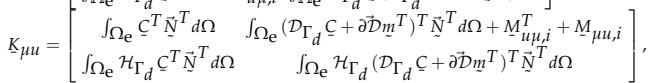

$$
\begin{aligned}
& \underline{M}_{u u, i}=h^{2} \int_{S_{d}} \vec{N} T_{i} \vec{N}^{T} d \Gamma=h^{2} \int_{S_{d}} \vec{N} T_{e, i} \vec{N}^{T} d \Gamma \\
& \underline{M}_{u \mu, i}=h \int_{S_{d}} \mathbb{N}^{+} \vec{n}^{+} \underline{m}^{T} d \Gamma \\
& M_{\mu \mu, i}=\int_{S_{d}} \frac{\partial m}{\partial \eta} \mid[\vec{u}]^{T} \vec{n}^{+}{ }^{k} K_{K_{d}} \frac{\partial m}{\partial \eta}{ }^{T} d \Gamma
\end{aligned}
$$

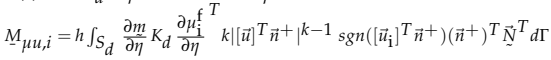

and

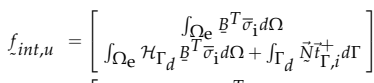

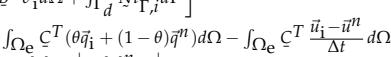

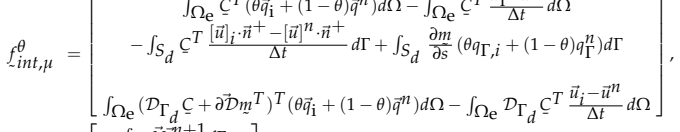

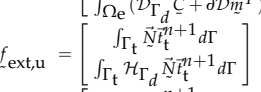

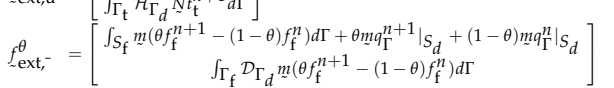

\section{References}

[1] Urban, J. P., and Roberts, S., 2003. "Degeneration of the intervertebral disc". Arthritis Res. Ther., 5(3), pp. 120-130.

[2] Adams, M. A., and Roughley, P. J., 2006. "What is intervertebral disc degeneration, and what causes it?". Spine, 31(18), pp. 2151-2161.

[3] Hutton, W. C., Ganey, T. M., Elmer, W. A., Kozlowska, E., Ugbo, J. L., Doh, E. S., and T. E. Whitesides, J., 2000. "Does long-term compressive loading on the intervertebral disc cause degeneration?". Spine, 25(23), pp. 2993-3004.

[4] Simunic, D. I., Broom, N. D., and Robertson, P. A., 2001. "Biomechanical factors influencing nuclear disruption of the intervertebral disc". Spine, 26(11), pp. 1223-1230. 
[5] Simunic, D. I., Robertson, P. A., and Broom, N. D., 2004. "Mechanically induced disruption of the healthy bovine intervertebral disc". Spine, 29(9), pp. 972-978.

[6] Callaghan, J. P., and McGill, S. M., 2001. “Intervertebral disc herniation: studies on a porcine model exposed to highly repetitive flexion/extension motion with compressive force". Clin. Biomech. (Bristol. , Avon. ), 16(1), pp. 28-37.

[7] Yu, C. Y., Tsai, K. H., Hu, W. P., Lin, R. M., Song, H. W., and Chang, G. L., 2003. "Geometric and morphological changes of the intervertebral disc under fatigue testing". Clin. Biomech. (Bristol. , Avon. ), 18(6), pp. S3-S9.

[8] Battie, M. C., Videman, T., and Parent, E., 2004. "Lumbar disc degeneration: epidemiology and genetic influences". Spine, 29(23), pp. 2679-2690.

[9] Videman, T., and Battie, M. C., 1999. "The influence of occupation on lumbar degeneration". Spine, 24(11), pp. 1164-1168.

[10] Wognum, S., Huyghe, J. M., and Baaijens, F. P., 2006. "Influence of osmotic pressure changes on the opening of existing cracks in 2 intervertebral disc models". Spine, 31(16), pp. 1783-1788.

[11] Huang, N. C., and Russell, S. G., 1985. "Hydraulic fracturing of a saturated porous-medium .1. general-theory". Theoretical and Applied Fracture Mechanics, 4(3), pp. 201-213.

[12] Rice, J. R., and Cleary, M. P., 1976. "Some basic stress diffusion solutions for fluid-saturated elastic porous media with compressible constituents". Rev. Geophys. and Space Phys., 14(2), pp. 227-241.

[13] Detournay, E., and Cheng, A. H. D., 1991. "Planestrain analysis of a stationary hydraulic fracture in a poroelastic medium". International Journal of Solids and Structures, 27(13), pp. 1645-1662.

[14] Emerman, S. H., Turcotte, D. L., and Spence, D. A., 1986. "Transport of magma and hydrothermal solutions by laminar and turbulent fluid fracture". Physics of the Earth and Planetary Interiors, 41(4), pp. 249-259.

[15] Boone, T. J., Ingraffea, A. R., and Roegiers, J. C., 1991. "Simulation of hydraulic fracture propagation in poroelastic rock with application to stress measurement techniques". International Journal of Rock Mechanics and Mining Sciences \& Geomechanics Abstracts, 28(1), pp. 1-14.

[16] Detournay, E., and Garagash, D. I., 2003. “The near-tip region of a fluid-driven fracture propagating in a permeable elastic solid". Journal of Fluid Mechanics, 494, pp. 1-32.

[17] Kfoury, M., Ababou, R., Noetinger, B., and Quintard, M., 2006. "Upscaling fractured heterogeneous media: Permeability and mass exchange coefficient". Journal of Applied Mechanics-Transactions of the Asme, 73(1), pp. 41-46.

[18] Dormieux, L., Kondo, D., and Ulm, F. J., 2006. “A micromechanical analysis of damage propagation in fluid-saturated cracked media". Comptes Rendus Mecanique, 334(7), pp. 440-446.

[19] Dugdale, D. S., 1960. "Yielding of steel sheets containing slits". Journal of the Mechanics and Physics of Solids, 8(2), pp. 100-104.

[20] Barenblatt, G. I., 1962. "The mathematical theory of equilibrium cracks in brittle fracture". Advances in Applied Mechanics(7), pp. 55-129.

[21] Simoni, L., and Secchi, S., 2003. "Cohesive fracture mechanics for a multi-phase porous medium". Engineering Computations, 20(5-6), pp. 675-698.

[22] Schrefler, B. A., Secchi, S., and Simoni, L., 2006. "On adaptive refinement techniques in multi-field problems including cohesive fracture". Computer Methods in Applied Mechanics and Engineering, 195(4-6), pp. 444-461.

[23] Secchi, S., Simoni, L., and Schrefler, B. A., 2007. "Mesh adaptation and transfer schemes for discrete fracture propagation in porous materials". International Journal for Numerical and Analytical Methods in Geomechanics, 31(2), pp. 331-345.

[24] Babuska, I., and Melenk, J. M., 1997. "The partition of unity method". International Journal for $\mathrm{Nu}$ merical Methods in Engineering, 40(4), pp. 727-758.

[25] Belytschko, T., and Black, T., 1999. "Elastic crack growth in finite elements with minimal remeshing". International Journal for Numerical Methods in Engineering, 45(5), pp. 601-620.

[26] Wells, G. N., 2001. "Discontinuous modelling of strain localisation and failure". PhD thesis, Delft University of Technology.

[27] Moes, N., and Belytschko, T., 2002. "Extended finite element method for cohesive crack growth". Engineering Fracture Mechanics, 69(7), pp. 813-833.

[28] Remmers, J. J. C., de Borst, R., and Needleman, A., 2003. "A cohesive segments method for the simulation of crack growth". Computational Mechanics, 31(1-2), pp. 69-77.

[29] Larsson, R., Runesson, K., and Ottosen, N. S., 1993. "Discontinuous displacement approximation for capturing plastic localization". International Journal for Numerical Methods in Engineering, 36(12), pp. 2087-2105.

[30] Larsson, J., and Larsson, R., 2000. "Localization analysis of a fluid-saturated elastoplastic porous medium using regularized discontinuities". Mechanics of Cohesive-Frictional Materials, 5(7), pp. 565-582.

[31] Armero, F., and Callari, C., 1999. "An analysis of strong discontinuities in a saturated poro-plastic solid". International Journal for Numerical Methods in Engineering, 46(10), pp. 1673-1698.

[32] Roels, S., Moonen, P., Proft, K. D., and Carmeliet, J., 2006. "A coupled discrete-continuum approach to simulate moisture effects on damage processes in porous materials". Computer Methods in Applied Mechanics and Engineering, 195(52), pp. 7139-7153.

[33] Al-Khoury, R., and Sluys, L. J., 2007. "A computa- 
tional model for fracturing porous media". International Journal for Numerical Methods in Engineering, 70(4), pp. 423-444.

[34] Rethore, J., de Borst, R., and Abellan, M. A., 2007. "A two-scale approach for fluid flow in fractured porous media". International Journal for Numerical Methods in Engineering, 71(7), pp. 780-800.

[35] de Borst, R., 2008. "Challenges in computational materials science: Multiple scales, multi-physics and evolving discontinuities". Computational Materials Science, 43(1), pp. 1-15.

[36] Lanir, Y., 1987. "Biorheology and fluid flux in swelling tissues .1. bicomponent theory for small deformations, including concentration effects". Biorheology, 24(2), pp. 173-187.

[37] Camacho, G. T., and Ortiz, M., 1996. "Computational modelling of impact damage in brittle materials". International Journal of Solids and Structures, 33(20-22), pp. 2899-2938.

[38] Vermeer, P. A., and Verruijt, A., 1981. "An accuracy condition for consolidation by finiteelements". International Journal for Numerical and Analytical Methods in Geomechanics, 5(1), pp. 1-14.

[39] Remmers, J. J. C., 2006. “Discontinuities in materials and structures. a unifying computational approach". PhD thesis, University of Technology Delft, The Netherlands.

[40] Remmers, J. J. C., de Borst, R., and Needleman, A., 2008. "The simulation of dynamic crack propagation using the cohesive segments method". Journal of the Mechanics and Physics of Solids, 56(1), pp. 7092.

[41] Zienkiewicz, O. C., Qu, S., Taylor, R. L., and Nakazawa, S., 1986. "The patch test for mixed formulations". International Journal for Numerical Methods in Engineering, 23(10), pp. 1873-1883. 